\title{
Maturation Asynchrony Present
}

National Cancer Institute

\section{Source}

National Cancer Institute. Maturation Asynchrony Present. NCI Thesaurus. Code C82930.

A morphologic finding indicating lack of synchronous maturation of the cytoplasm and the nucleus. 